\title{
Better Redistribution with Inefficient Allocation in Multi-Unit Auctions with Unit Demand
}

\author{
Mingyu Guo \\ Duke University \\ Department of Computer Science \\ Durham, NC, USA \\ mingyu@cs.duke.edu
}

\author{
Vincent Conitzer * \\ Duke University \\ Department of Computer Science \\ Durham, NC, USA \\ conitzer@cs.duke.edu
}

\begin{abstract}
For the problem of allocating one or more items among a group of competing agents, the Vickrey-Clarke-Groves (VCG) mechanism is strategy-proof and efficient. However, the VCG mechanism is not strongly budget balanced: in general, value flows out of the system of agents in the form of VCG payments, which reduces the agents' utilities. In many settings, the objective is to maximize the sum of the agents' utilities (taking payments into account). For this purpose, several VCG redistribution mechanisms have been proposed that redistribute a large fraction of the VCG payments back to the agents, in a way that maintains strategy-proofness and the non-deficit property. Unfortunately, sometimes even the best VCG redistribution mechanism fails to redistribute a substantial fraction of the VCG payments. This results in a low total utility for the agents, even though the items are allocated efficiently. In this paper, we study strategyproof allocation mechanisms that do not always allocate the items efficiently. It turns out that by allocating inefficiently, more payment can sometimes be redistributed, so that the net effect is an increase in the sum of the agents' utilities.

Our objective is to design mechanisms that are competitive with the omnipotent perfect allocation in terms of the agents' total utility. We define linear allocation mechanisms. We propose an optimization model for simultaneously finding an allocation mechanism and a payment redistribution rule which together are optimal, given that the allocation mechanism is required to be either one of, or a mixture of, a finite set of specified linear allocation mechanisms. Finally, we propose several specific (linear) mechanisms that are based on burning items, excluding agents, and (most generally) partitioning the items and agents into groups. We show or conjecture that these mechanisms are optimal among various classes of mechanisms.
\end{abstract}

${ }^{*}$ Conitzer is supported by an Alfred P. Sloan Research Fellowship.

Permission to make digital or hard copies of all or part of this work for personal or classroom use is granted without fee provided that copies are not made or distributed for profit or commercial advantage and that copies bear this notice and the full citation on the first page. To copy otherwise, to republish, to post on servers or to redistribute to lists, requires prior specific permission and/or a fee.

EC'08, July 8-12, 2008, Chicago, Illinois, USA.

Copyright 2008 ACM 978-1-60558-169-9/08/07 ...\$5.00.

\section{Categories and Subject Descriptors}

J.4 [Computer Applications]: Social and Behavioral Sciences-Economics; I.2.11 [Distributed Artificial Intelligence]: Multiagent Systems

\section{General Terms}

Algorithms, Economics, Theory

\section{Keywords}

Mechanism design, Vickrey-Clarke-Groves mechanism, payment redistribution, welfare maximization, inefficient allocation

\section{INTRODUCTION}

Many problems in electronic commerce involve resource allocation. For the problem of allocating one or more items among a group of competing agents, the well-known VickreyClarke-Groves (VCG) mechanism $[20,5,11]$ is strategyproof and efficient. ${ }^{1}$ That is, under the VCG mechanism, it is a dominant strategy for the agents to report their true valuations for the items, and the mechanism allocates the items in a way that maximizes the sum of the agents' valuations. In the context of resource allocation, the VCG mechanism is also weakly budget balanced (aka. it satisfies the non-deficit property): the sum of the agents' payments is nonnegative (in fact, each individual agent's payment is nonnegative). However, the VCG mechanism is not strongly budget balanced, that is, the agents' payments do not sum to zero. Hence, in general, value flows out of the system of agents in the form of VCG payments, which reduces the agents' utilities. Actually, for sufficiently general settings, no mechanism satisfies strategy-proofness, efficiency and strong budget balance at the same time $[16,10,9,18]$. (By sacrificing one of these properties, the others can be achieved $[2,7,19$, $6]$.

In many cases, the objective of the allocation process is to maximize the welfare of the agents, that is, the sum of the agents' utilities, taking payments into account. For this purpose, several $V C G$ redistribution mechanisms have been proposed. These mechanisms first allocate the items efficiently and charge the VCG payments. Then, a large fraction of the VCG revenue is redistributed back to the agents, in a way that maintains the desirable properties of the origi-

\footnotetext{
${ }^{1}$ As is often done, we use "the VCG mechanism" to refer to the Clarke mechanism, and not to any other Groves mechanism.
} 
nal VCG mechanism, including strategy-proofness, the nondeficit property, and (sometimes) individual rationality [4, $12,17,14,13]$. (A mechanism is individually rational if participating in the mechanism never makes an agent worse off.) However, in some cases, even the best redistribution mechanism fails to redistribute a substantial amount of the VCG revenue. That is, even though the VCG redistribution mechanisms maximize efficiency (the sum of the agents' valuations), the total welfare (the sum of the agents' utilities, taking payments into account) can be very low (in fact, zero), as a result of poor redistribution. Still, the previously proposed VCG redistribution mechanisms are optimal in various senses - but only under the constraint that allocation is efficient.

In this paper, we consider the natural next step of allowing for inefficient allocation. It turns out that even though inefficient allocation reduces efficiency, it sometimes allows for greater redistributions, so that the net effect is an increase in the sum of the agents' utilities. Moulin [17] already provided an example where inefficient allocation can lead to better results, but left a more thorough investigation for future research. As we will see, the example mechanism that he proposed will turn out to be useful for us.

In Section 2, we cover some basic definitions that we will use in this paper. In Section 3, we briefly review the worstcase optimal VCG redistribution mechanism $[12,17]$. In Section 4 , we define a class of allocation mechanisms that we call linear allocation mechanisms, and propose an optimization model for simultaneously finding an allocation mechanism and a payment redistribution rule which together are optimal, given that the allocation mechanism is required to be either one of, or a mixture over, a finite set of specified linear allocation mechanisms. In Section 5 to Section 6, we propose several specific mechanisms that are based on burning items, excluding agents, and (most generally) partitioning the items and agents into groups. We show or conjecture that these mechanisms are optimal among various classes of mechanisms.

\section{PROBLEM DESCRIPTION}

We will restrict our attention to multi-unit auctions with unit demand in this paper. In such auctions, multiple indistinguishable units are for sale at the same time, and each agent is interested in one and only one unit.

Let the number of units be $m$. ( $m=1$ corresponds to a single-item auction.) Let the number of agents be $n$. We consider only cases where $n>m$. (Otherwise, it is clearly optimal to give every agent a unit and charge nothing.) For the $i$ th agent, we denote her reported value (bid) for winning one unit by $\hat{v}_{i}$, and we denote her true value for winning one unit by $v_{i}$. In a strategy-proof mechanism, it is optimal for each agent to set $\hat{v}_{i}=v_{i}$, and we only study strategy-proof mechanisms in this paper. Hence, from here on, we use the $v_{i}$ to denote both the reported values (bids) and the true values. Without losing generality, we assume that $v_{1} \geq v_{2} \geq$ $\ldots \geq v_{n} \geq 0$. A bid profile is a vector $V=\left(v_{1}, v_{2}, \ldots, v_{n}\right)$.

Let $M$ be a strategy-proof allocation mechanism. (In this paper, we use "allocation mechanism" to refer to the mechanism before redistribution - for example, in a VCG redistribution mechanism, the VCG mechanism is the allocation mechanism, whereas the complete mechanism also includes the redistributions.) $M$ does not need to be deterministic: in general, $M$ can be a probability mixture over $t$ deterministic mechanisms $M_{1}, \ldots, M_{t}$. (When $t=1, M$ is deterministic.) With probability $p_{i}$, mechanism $M_{i}$ is chosen $\left(\sum_{i=1}^{t} p_{i}=1\right)$.

For each bid profile $V$, we define $U_{M}(V)$ to be the total efficiency (sum of obtained valuations) that results under $M$ for $V$ (this does not take payments into account). We have $U_{M}(V)=\sum_{i=1}^{t} p_{i} U_{M_{i}}(V)$. Similarly, let $P_{M}(V)$ be the total revenue (sum of the agents' payments) that results under $M$ for $V$. We have $P_{M}(V)=\sum_{i=1}^{t} p_{i} P_{M_{i}}(V)$.

For multi-unit auctions with unit demand, the VCG mechanism is just the $(m+1)$ th price auction: the agents with the $m$ highest bids each win one unit and each pay the value of the $(m+1)$ th-highest bid. Hence, if $M$ is the VCG mechanism, $U_{M}(V)=\sum_{i=1}^{m} v_{i}$ and $P_{M}(V)=m v_{m+1}$.

Given a strategy-proof allocation mechanism $M$ and a bid profile $V$, without redistribution, the agents' welfare under $M$ equals $U_{M}(V)-P_{M}(V)$. (We assume quasilinear preferences throughout the paper, so that utilities and payments have the same units.) The welfare can potentially be increased by introducing redistribution payments. We require that the redistribution payment to each agent is independent of her own bid, so that the mechanism will remain strategy-proof. That is, agent $i$ receives a redistribution payment $R\left(V_{\sim i}\right)$, where $V_{\sim i}$ is the bid profile without $v_{i}$ $\left(v_{1}, \ldots, v_{i-1}, v_{i+1}, \ldots, v_{n}\right)$, and $R$ is any real-valued function.

Not all redistribution functions $(R)$ are feasible. For a redistribution mechanism to be feasible, we require two additional properties. First, we require that $P_{M}(V) \geq \sum_{i=1}^{n} R\left(V_{\sim i}\right)$ for all $V$. That is, the mechanism must satisfy the nondeficit property: the total redistribution should never exceed the revenue collected by $M$. Otherwise, we need external funds to subsidize the mechanism.

We also require that the mechanism be (ex-post) individually rational: if $M$ is deterministic, then for any bid profile $V$, every agent's utility after redistribution must be nonnegative. If $M$ is not deterministic, then for any bid profile $V$, every agent's expected utility after redistribution must be nonnegative.

With redistribution, for a bid profile $V$, the agents' welfare is $U_{M}(V)-P_{M}(V)+\sum_{i=1}^{n} R\left(V_{\sim i}\right)$. Our goal is to find a strategy-proof allocation mechanism $M$ and a redistribution function $R$ that are feasible and maximize this expression. However, this is not a well-defined objective, because the value of this expression depends on $V$. It could be that one choice of $M$ and $R$ maximizes the expression for some $V$, while another choice of $M$ and $R$ maximizes the expression for another $V$. In this paper, we pursue a worst-case analysis approach. Such approaches have been commonly used in the literature on redistribution mechanisms $[12,17]^{2}$ as well as the literature on digital goods auctions $[1,8,15]$. Specifically, consider an omnipotent perfect allocation mechanism that magically identifies the agents with the $m$ highest true valuations, without asking for their bids, and allocates the units to these agents at no charge. Clearly this mechanism obtains the largest welfare that we could hope for (without deficits). Our objective is to design mechanisms that are competitive with this perfect allocation mechanism. We say a redistribution mechanism $(M, R)$ is $\alpha$-competitive with the

\footnotetext{
${ }^{2}$ However, other objectives have been pursued as well, for example, maximizing the expected redistribution with respect to a prior [13], or making sure that there is no other feasible mechanism that always redistributes at least as much to every agent [14].
} 
perfect mechanism if the agents' welfare under $(M, R)$ is at least $\alpha \sum_{i=1}^{m} v_{i}$, for all bid profiles $V$. ( $\sum_{i=1}^{m} v_{i}$ is the agents' welfare under the perfect mechanism.) Our objective is to find the redistribution mechanism $(M, R)$ that is the most competitive, that is, that maximizes $\alpha$, while satisfying the individual rationality and non-deficit properties.

\section{VCG REDISTRIBUTION MECHANISMS}

In this section, we first review the worst-case optimal VCG redistribution mechanism. Then, to motivate the rest of the paper, we construct an example feasible strategy-proof mechanism that allocates inefficiently and has a higher competitive ratio (with the perfect mechanism) than all feasible strategy-proof mechanisms that always maximize efficiency.

In previous work [12], we characterized the worst-case optimal (WCO) VCG redistribution mechanism, which maximizes the fraction of VCG revenue that is redistributed in the worst case, among all VCG redistribution mechanisms that are individually rational and satisfy the nondeficit property. The WCO mechanism uses the following redistribution function:

$$
\begin{aligned}
& R\left(V_{\sim j}\right)=\sum_{i=m+1}^{n-1} c_{i} V_{\sim j}(i) \\
& c_{i}=\frac{(-1)^{i+m-1}(n-m)\left(\begin{array}{c}
n-1 \\
m-1
\end{array}\right)}{i \sum_{j=m}^{n-1}\left(\begin{array}{c}
n-1 \\
j
\end{array}\right)} \frac{1}{\left(\begin{array}{c}
n-1 \\
i
\end{array}\right)} \sum_{j=i}^{n-1}\left(\begin{array}{c}
n-1 \\
j
\end{array}\right)
\end{aligned}
$$

Here, $V_{\sim j}(i)$ is the $i$ th-highest bid among $V_{\sim j}$ (the bids other than $j$ 's bid).

Around the same time, Moulin [17] independently characterized the same mechanism, based on a different objective. ${ }^{3}$ Specifically, Moulin tries to minimize the worst-case ratio between the VCG revenue that fails to be redistributed and the total efficiency, also among all VCG redistribution mechanisms that are individually rational and satisfy the non-deficit property. In fact, Moulin's characterization of WCO implies that the WCO mechanism has the highest competitive ratio (in the sense of this paper) among all (efficient) VCG redistribution mechanisms with the required properties.

Claim 1. The WCO mechanism has the highest competitive ratio $\alpha$ with the perfect allocation mechanism, among all (efficient) VCG redistribution mechanisms that are individually rational and satisfy the non-deficit property.

Proof. Moulin's objective is to find a redistribution function $R$ that minimizes $\frac{P_{M}(V)-\sum_{i=1}^{n} R\left(V_{\sim i}\right)}{U_{M}(V)}$ in the worst case. This is equivalent to maximizing $\frac{U_{M}(V)-P_{M}(V)+\sum_{i=1}^{n} R\left(V_{\sim i}\right)}{U_{M}(V)}$ in the worst case. The denominator equals the agents' welfare under the perfect mechanism, because $M$ is by definition efficient (in any VCG redistribution mechanism, units are allocated according to the original VCG mechanism). Therefore, Moulin's objective is equivalent to maximizing the competitive ratio with the perfect mechanism.

Given the number of agents $n$ and the number of units $m$ $(n>m)$, the WCO mechanism's competitive ratio $\alpha_{W C O}(n, m)$ is characterized by the following equation:

\footnotetext{
${ }^{3}$ The objectives only result in the same mechanism if individual rationality is required. If it is not required, then the objective we used still results in the WCO mechanism, but Moulin's does not.
}

$$
\alpha_{W C O}(n, m)=1-\frac{\left(\begin{array}{c}
n-1 \\
m
\end{array}\right)}{\sum_{j=m}^{n-1}\left(\begin{array}{c}
n-1 \\
j
\end{array}\right)}
$$

When $n=3$ and $m=2$, the WCO mechanism is not competitive at all: $\alpha_{W C O}(3,2)=0$. In contrast, the following simple mechanism that allocates inefficiently is somewhat competitive:

- Burn (throw away) one unit.

- Allocate the remaining unit according to the WCO mechanism for $n=3$ and $m=1$.

The new mechanism is feasible and strategy-proof because it is equivalent to the WCO mechanism for $n=3$ and $m=1$. It is not efficient because one unit is burned. Since $\alpha_{W C O}(3,1)=\frac{1}{3}$, the new mechanism is $\frac{1}{3}$-competitive with the perfect allocation mechanism for one unit $(m=1)$. That is, the new mechanism guarantees a welfare of $\frac{1}{3} v_{1}$ for any bid profile $V$. Since $v_{1} \geq v_{2}$, it also guarantees a total utility of $\frac{1}{6}\left(v_{1}+v_{2}\right)$ for all bid profiles. Hence, the competitive ratio of the new mechanism with the perfect allocation mechanism for two units $(m=2)$ is at least $\frac{1}{6}$. That is, this new mechanism has a higher competitive ratio than any VCG redistribution mechanism (any feasible strategyproof mechanism that allocates the units efficiently). So, ironically, in some cases, the agents are happier if one unit is burned. Motivated by this example, in the rest of the paper, we study mechanisms that allocate inefficiently (and in Section 5 , we specifically study mechanisms that are based on burning units).

\section{LINEAR ALLOCATION MECHANISMS}

In this section, we define a class of mechanisms that we call linear allocation mechanisms. We then provide a general technique for finding the optimal redistribution function for any given linear allocation mechanism. We also show how to simultaneously find the optimal linear allocation mechanism and the corresponding redistribution function, given that the allocation mechanism is required to be one of, or a mixture over, a finite set of specified linear allocation mechanisms.

Definition 1. A (strategy-proof) allocation mechanism $M$ is linear if the following two conditions are satisfied:

- (linearity) $U_{M}(V)$ and $P_{M}(V)$ are linear combinations of the $v_{i}$.

- (normalized individual rationality) $M$ is individually rational, and an agent's payment is always 0 if her bid is 0 .

Example 1. The VCG mechanism is linear, for the following reasons. In the VCG mechanism, the agents with the highest $m$ bids each win one unit and each pay the value of the $(m+1)$ th-highest bid. That is, for any bid profile $V=$ $\left(v_{1}, v_{2}, \ldots, v_{n}\right), U_{M}(V)=\sum_{i=1}^{m} v_{i}$ and $P_{M}(V)=m v_{m+1}$, which are both linear. ${ }^{4}$ The normalized individual rationality condition is also satisfied by the VCG mechanism. Under

${ }^{4}$ We emphasize that the linearity depends on the fact that the bids are sorted. In fact, if we increase the $(m+1)$ thhighest bid, then the revenue will increase, but only up to the point where the bid equals the $m$ th-highest bid; if we increase the bid further, the revenue will not change. So in this sense, the VCG mechanism is not linear in the bids, but this is not the type of linearity that is used in the definition. 
the VCG mechanism, the payment from an agent is always less than or equal to her own bid, and is never negative. When an agent's bid is 0 , her payment must be 0 .

Example 2. The random allocation mechanism in which the winners are picked uniformly at random (without replacement), and there are no payments, is linear, for the following reasons. Under this mechanism, for any bid profile $V, U_{M}(V)=\frac{m}{n} \sum_{i=1}^{n} v_{i}$, and $P_{M}(V)=0$, which are both linear. The normalized individual rationality condition is also satisfied.

Claim 2. Any probability mixture over linear allocation mechanisms is also linear.

Proof. Let $M$ be a mixture over $t$ linear allocation mechanisms $M_{1}, M_{2}, \ldots, M_{t}$, where $M_{i}$ is chosen with probability $p_{i}$. We have $U_{M}(V)=\sum_{i=1}^{t} p_{i} U_{M_{i}}(V)$ and $P_{M}(V)=$ $\sum_{i=1}^{t} p_{i} P_{M_{i}}(V)$, which are both linear, because for any $i$, $U_{M_{i}}$ and $P_{M_{i}}$ are linear. Normalized individual rationality also holds: because $M_{1}, M_{2}, \ldots, M_{t}$ are all individually rational, any mixture over them is also individually rational. If an agent's bid is 0 , then for all $i$, her payment under $M_{i}$ is 0 . This implies that her payment under any mixture over the $M_{i}$ is also 0 .

Given the number of agents $n$, the number of units $m$, and a linear allocation mechanism $M$, the following optimization model can be used to find an optimal redistribution function $R$, so that the resulting mechanism $(M, R)$ has the highest competitive ratio. That is, we are computing the optimal redistribution function for a fixed allocation mechanism.

\begin{tabular}{|l|}
\hline Variable function: $R:[0, \infty)^{n-1} \rightarrow \mathbb{R}$ \\
Variable: $\alpha$ \\
Maximize $\alpha$ \\
Subject to: \\
For every bid profile $V=\left(v_{1}, v_{2}, \ldots, v_{n}\right)$ \\
with $v_{1} \geq v_{2} \geq \ldots \geq v_{n} \geq 0$ \\
$R\left(V_{\sim n}\right) \geq 0$ (individual rationality) \\
$P_{M}(V) \geq \sum_{i=1}^{n} R\left(V_{\sim i}\right)$ (non-deficit) \\
$U_{M}(V)-P_{M}(V)+\sum_{i=1}^{n} R\left(V_{\sim i}\right) \geq \alpha \sum_{i=1}^{m} v_{i}$ \\
$($ competitive ratio constraint)
\end{tabular}

Because $M$ is linear, it satisfies the normalized individual rationality condition. Hence, if the agent with the lowest bid bids 0 , her payment under $M$ must be 0 . Such an agent's utility is 0 when there is no redistribution, regardless of whether she wins a unit or not. With redistribution, such an agent's utility is just her redistribution $R\left(V_{\sim n}\right)$. Therefore, for the resulting mechanism $(M, R)$ to satisfy the individual rationality constraint, it is necessary that $R\left(V_{\sim n}\right) \geq 0$ for all $V_{\sim n}$. Since $R\left(V_{\sim n}\right)$ does not depend on the value of $v_{n}$, equivalently, it is necessary that $R\left(V_{\sim n}\right) \geq 0$ for all $V$. Conversely, if $R\left(V_{\sim_{n}}\right) \geq 0$ for all $V$, then the function $R$ is always nonnegative, because for any $x_{1} \geq x_{2} \geq \ldots \geq x_{n-1}$ there exists a $V$ such that $V_{\sim n}=\left(x_{1}, x_{2}, \ldots, x_{n-1}\right)$. This implies that $R\left(V_{\sim n}\right) \geq 0$ for all $V$ is also a sufficient condition for individual rationality, because $M$ is individually rational without redistribution and nonnegative redistribution never decreases an agent's utility. This is why the individual rationality constraint can be written as $R\left(V_{\sim n}\right) \geq 0$ for all $V$.

Now, suppose that the allocation mechanism is not fixed; specifically, suppose that we need to choose one mechanism
$M$ from a set of $t$ linear allocation mechanisms $\left\{M_{1}, \ldots, M_{t}\right\}$, so that $M$, coupled with a corresponding optimal redistribution function, has the highest competitive ratio. Then the optimization model becomes:

Variable function: $R:[0, \infty)^{n-1} \rightarrow \mathbb{R}$
Variable: $\alpha$
Binary variables: $p_{1}, p_{2}, \ldots, p_{t}$
Maximize $\alpha$
Subject to:
For every bid profile $V=\left(v_{1}, v_{2}, \ldots, v_{n}\right)$
with $v_{1} \geq v_{2} \geq \ldots \geq v_{n} \geq 0$
$R\left(V_{\sim n}\right) \geq 0$ (individual rationality)
$\sum_{j=1}^{t} p_{j} P_{M_{j}}(V) \geq \sum_{i=1}^{n} R\left(V_{\sim i}\right)($ non-deficit)
$\sum_{j=1}^{t} p_{j} U_{M_{j}}(V)-\sum_{j=1}^{t} p_{j} P_{M_{j}}(V)+\sum_{i=1}^{n} R\left(V_{\sim i}\right)$
$\geq \alpha \sum_{i=1}^{m} v_{i}($ competitive ratio constraint)
$\sum_{j=1}^{t} p_{j}=1$

It might not be clear, at first glance, why we would want to introduce the binary variables $p_{j}$, rather than just solve the original model $t$ times. The reason is that if we change the $p_{j}$ into continuous variables ranging from 0 to 1 , then the modified model optimizes for the best allocation mechanism among mechanisms that are mixtures over $\left\{M_{1}, M_{2}, \ldots, M_{t}\right\}$ (and simultaneously, it optimizes the corresponding redistribution function). We call the above optimization model in which the $p_{j}$ are binary the discrete model (DM), and we call the modified optimization model the continuous model (CM).

DM and CM both optimize over functions, not just variables. However, as it turns out, an optimal solution can be found by means of a linear program (for CM) or a mixed integer program (for DM). The linear/mixed integer program can be solved directly, using any solver.

The constraints of DM and CM must be satisfied for any bid profile $V=\left(v_{1}, v_{2}, \ldots, v_{n}\right)$ with $v_{1} \geq v_{2} \geq \ldots \geq v_{n} \geq 0$. We use $z \in\{0, \ldots, n\}$ to denote the bid profile in which the highest $z$ bids are 1 and the remaining bids are 0 . If we only require that the constraints be satisfied for bid profiles from 0 to $n$, then the objective value should be greater than or equal to the original objective value. The relaxed optimization models (UCM and UDM, for "upper bounding continuous/discrete model") are:

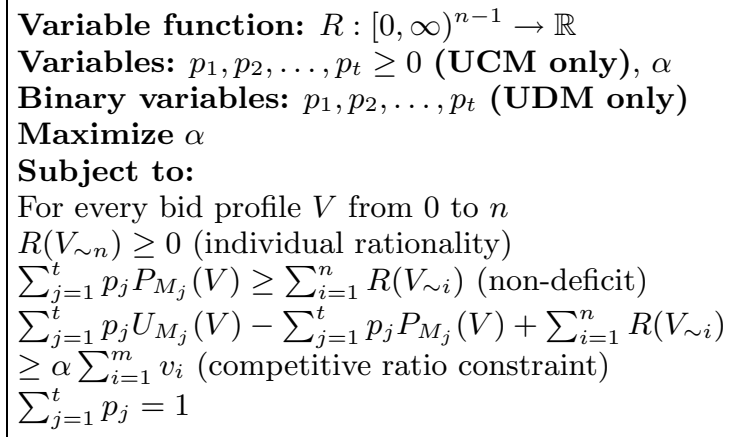

Effectively, UCM is a linear program and UDM is a mixed integer program. If $V=z$, then $V_{\sim i}$ (the bids other than $i$ 's own bid) contains $z$ copies of 1 and $n-1-z$ copies of 0 for $i>z$, and $V_{\sim i}$ contains $z-1$ copies of 1 and $n-z$ copies of 0 for $i \leq z$. Let us denote $R\left(V_{\sim i}\right)$ by $R_{x}$ if $V_{\sim i}$ contains $x$ copies of $1(0 \leq x \leq n-1)$. Then, the $n$ variables 
$R_{0}, R_{1}, \ldots, R_{n-1}$ specify everything about the redistribution function that affects the UDM/UCM programs; thus, they are the only variables that we need, in addition to the $p_{j}$ and $\alpha$. The $P_{M_{j}}(V)$ and $U_{M_{j}}(V)$ are constants that we need to evaluate for $V$ from 0 to $n$. The constraints and the objective function are all linear. This results in the following linear/mixed integer program:

\begin{tabular}{l} 
Variables: $p_{1}, p_{2}, \ldots, p_{t} \geq 0$ (UCM only) \\
$\alpha, R_{0}, R_{1}, \ldots, R_{n-1}$ \\
Binary variables: $p_{1}, p_{2}, \ldots, p_{t}$ (UDM only) \\
Maximize $\alpha$ \\
Subject to: \\
$\sum_{j=1}^{t} p_{j}=1$ \\
$R_{0}=0, R_{x} \geq 0$ for $1 \leq x \leq n-1$ \\
$\sum_{j=1}^{t} p_{j} P_{M_{j}}(x) \geq x R_{x-1}+(n-x) R_{x}$ for $1 \leq x \leq n$ \\
$\sum_{j=1}^{t} p_{j} U_{M_{j}}(x)-\sum_{j=1}^{t} p_{j} P_{M_{j}}(x)+x R_{x-1}+(n-x) R_{x}$ \\
$\geq \alpha \min \{x, m\}$ for $1 \leq x \leq n$ \\
\hline
\end{tabular}

Let $\alpha_{C}^{*}, R_{C, 0}^{*}, R_{C, 1}^{*}, \ldots, R_{C, n-1}^{*}$, and $p_{C, 1}^{*}, p_{C, 2}^{*}, \ldots, p_{C, t}^{*}$ denote an optimal solution to UCM; similarly, let $\alpha_{D}^{*}$, $R_{D, 0}^{*}, R_{D, 1}^{*}, \ldots, R_{D, n-1}^{*}$, and $p_{D, 1}^{*}, p_{D, 2}^{*}, \ldots, p_{D, t}^{*}$ denote an optimal solution to UDM. We know that $\alpha_{C}^{*}\left(\alpha_{D}^{*}\right)$ is an upper bound on the competitive ratio that can be obtained in the continuous (discrete) case; we will show that, in fact, $\alpha_{C}^{*}\left(\alpha_{D}^{*}\right)$ can be obtained, so that it is the optimal competitive ratio. The following theorem shows how to convert the optimal solution to UCM (UDM) into a redistribution mechanism that is defined for all $V$ and that obtains competitive ratio $\alpha_{C}^{*}\left(\alpha_{D}^{*}\right)$.

TheOREM 1. The optimal objective value for $C M$ (DM) equals $\alpha_{C}^{*}\left(\alpha_{D}^{*}\right)$. For DM, an optimal allocation mechanism is $M_{j}$, where $j$ is the (only) index that satisfies $p_{D, j}^{*}=1$. For $C M$, an optimal allocation mechanism is the mixture over $M_{1}, M_{2}, \ldots, M_{t}$ where $M_{j}$ is chosen with probability $p_{C, j}^{*}$.

An optimal redistribution function $R_{C}$ can be obtained from the $R_{C, x}^{*}$ as follows: for any $V$ and any $i$,

$$
R_{C}\left(V_{\sim i}\right)=R_{C, 0}^{*} V_{\sim i}(1)+\sum_{x=1}^{n-1}\left(R_{C, x}^{*}-R_{C, x-1}^{*}\right) V_{\sim i}(x)
$$

Here, $V_{\sim i}(x)$ is the $x$ th-highest bid among bids other than $i$ 's own bid. An optimal redistribution function $R_{D}$ is defined similarly.

We note that when $V_{\sim i}$ consists of $z$ ones and $n-z-1$ zeroes, we have $R_{C}\left(V_{\sim i}\right)=R_{C, 0}^{*}+\sum_{x=1}^{z}\left(R_{C, x}^{*}-R_{C, x-1}^{*}\right)=$ $R_{C, z}^{*}$ (if $\left.z=0, R_{C}\left(V_{\sim i}\right)=0=R_{C, 0}^{*}\right)$. (In a sense, $R$ is an interpolation of these values.) Before proving the theorem, we give the following lemma. A similar lemma appeared in Guo and Conitzer [12].

Lemma 1. When the $c_{i}$ do not depend on the $x_{i}$, the following two systems of inequalities are equivalent:

(a) $c_{1} x_{1}+c_{2} x_{2}+\ldots+c_{s} x_{s} \geq 0$ for all $x_{1} \geq x_{2} \geq \ldots \geq$ $x_{s} \geq 0$.

(b) $c_{1} x_{1}+c_{2} x_{2}+\ldots+c_{s} x_{s} \geq 0$ for all $x_{1} \geq x_{2} \geq \ldots \geq x_{s}$, where each $x_{i} \in\{0,1\}$.

Proof. (a) $\Rightarrow$ (b) is trivial. We now prove (b) $\Rightarrow$ (a). (b) implies that $\sum_{i=1}^{j} c_{i} \geq 0$ for all $1 \leq j \leq s$. We have $c_{1} x_{1}+c_{2} x_{2}+\ldots+c_{s} x_{s}=\sum_{j=1}^{s-1}\left(\sum_{i=1}^{j} c_{i}\right)\left(x_{j}-x_{j+1}\right)+$ $\left(\sum_{i=1}^{s} c_{i}\right) x_{s}$. For all $x_{1} \geq x_{2} \geq \ldots \geq x_{s} \geq 0$, each term of the above expression is nonnegative, hence the whole expression is nonnegative. So (b) $\Rightarrow$ (a).

Now we are ready to prove Theorem 1.

Proof. We only need to prove that the solution described in the theorem is a feasible solution for CM (DM). (We emphasize that feasibility also entails obtaining the competitive ratio $\alpha_{C}^{*}\left(\alpha_{D}^{*}\right)$ everywhere.) Because it is feasible, it is also optimal, because $\alpha_{C}^{*}\left(\alpha_{D}^{*}\right)$ is an upper bound on CM (DM).

In the proposed solution, we have $R_{C}\left(V_{\sim i}\right)=R_{C, 0}^{*} V_{\sim i}(1)+$ $\sum_{x=1}^{n-1}\left(R_{C, x}^{*}-R_{C, x-1}^{*}\right) V_{\sim i}(x)$. For specific $i$ and $x$, when $x<i, V_{\sim i}(x)=v_{x}$, and when $x \geq i, V_{\sim i}(x)=v_{x+1}$. (We recall that $V_{\sim i}(x)$ is the $x$ th-highest bid among bids other than $i$ 's own bid.) Hence, $R_{C}\left(V_{\sim i}\right)$ is linear in $v_{1}, v_{2}, \ldots, v_{n}$, where the coefficients are determined by the constants $R_{C, x}^{*}$ (we have similar results for $R_{D}$ ). For all $1 \leq j \leq t, U_{M_{j}}$ and $P_{M_{j}}$ are both linear in $v_{1}, v_{2}, \ldots, v_{n}$ by the linearity assumption. So for all of the constraints in CM (DM), with the exception of the probability constraint, each side of the inequality is a linear combination of the $v_{i}$.

We need to prove that these constraints are satisfied for all $v_{1} \geq v_{2} \geq \ldots \geq v_{n} \geq 0$. By Lemma 1 , we only need them be satisfied for all $v_{1} \geq v_{2} \geq \ldots \geq v_{n} \geq 0$ where the $v_{i}$ are binary variables. That is, we only need them be satisfied for the bid profiles $V$ from 0 to $n$. But for these $V$, the constraints of CM (DM) are identical with the constraints of UCM (UDM), because, as we already noted, the function $R_{C}\left(R_{D}\right)$ that we have defined coincides with the $R_{C, z}^{*}\left(R_{D, z}^{*}\right)$ on these $V$.

Using Theorem 1, given the number of agents $n$ and the number of units $m$, we can find the optimal allocation mechanism $M$, and simultaneously, a corresponding optimal redistribution function $R$, so that the resulting mechanism $(M, R)$ maximizes the competitive ratio-under the constraint that $M$ must be one of, or a mixture over, a finite set of specific linear allocation mechanisms $M_{1}, M_{2}, \ldots, M_{t}$.

\section{BURNING UNITS}

In this section, we study allocation mechanisms that are based on (sometimes) burning units. As the example in Section 3 showed, in some cases we can achieve a higher competitive ratio by burning units than by using the most competitive mechanism that is feasible, strategy-proof and efficient (the WCO mechanism).

We start by characterizing a set of mechanisms based on the idea of burning units. First, we construct $m$ allocation mechanisms that are based on burning a deterministic number of units. Let $M_{i}(i=1 \ldots m)$ be the allocation mechanism in which $m-i$ units are burned, and the remaining $i$ units are allocated efficiently according to the VCG mechanism. $M_{m}$ is just the original VCG mechanism. We note that it makes no sense to burn all units, hence $i>0$. We call the $M_{i}$ deterministic burning allocation mechanisms. We can also construct allocation mechanisms in which a random number of units are burned, by randomizing over the $M_{i}$. Let $M$ be a mixture of the $M_{i}$, where mechanism $M_{i}$ is chosen with probability $p_{i}$. That is, $M$ is the mechanism in which with probability $p_{i}$, exactly $m-i$ units are burned. (If $p_{i}=1$ for some $i$, then $M$ is just $M_{i}$.) We call such mixtures over the $M_{i}$ randomized burning allocation mechanisms. 
The deterministic burning allocation mechanisms are strategy-proof, because the remaining units are allocated according to the VCG mechanism, which is strategy-proof. It follows that the randomized burning allocation mechanisms are also strategy-proof. Also, the deterministic burning allocation mechanisms are linear. When there are $i$ units remaining $\left(M_{i}\right)$, the agents with the $i$ highest bids each win one unit, and each pay the value of the $(i+1)$-th highest bid. That is, for any bid profile $V=\left(v_{1}, v_{2}, \ldots, v_{n}\right)$, $U_{M_{i}}(V)=\sum_{j=1}^{i} v_{j}$ and $P_{M_{i}}(V)=i v_{i+1}$. Both $U_{M_{i}}$ and $P_{M_{i}}$ are linear in the $v_{i}$. The normalized individual rationality condition is also satisfied. Therefore, the deterministic burning allocation mechanisms are linear. By Claim 2, we also have that the randomized burning allocation mechanisms are linear.

Using Theorem 1, we can find an optimal allocation mechanism $M$, and a corresponding optimal redistribution function $R$, so that $(M, R)$ maximizes the competitive ratio, given that $M$ is one of the deterministic burning allocation mechanisms, or $M$ is a randomized burning allocation mechanism.

In the following table, we present the results for different numbers of agents and different numbers of units. The second column $\left(\alpha_{D}^{*}\right)$ gives the optimal competitive ratio among all feasible mechanisms $(M, R)$ where $M$ is one of the deterministic burning allocation mechanisms. The integers in the third column are the number of units burned in the optimal mechanism that corresponds to $\alpha_{D}^{*}$. The fourth column $\left(\alpha_{C}^{*}\right)$ is the optimal competitive ratio among all feasible mechanisms $(M, R)$ where $M$ is a randomized burning allocation mechanism. The values in the fifth column are the probabilities of having one unit burned in the optimal mechanism that corresponds to $\alpha_{C}^{*}$. (It turns out that in the optimal mechanism, either exactly one unit is burned with a certain probability, or nothing is burned.) Finally, as a benchmark, the sixth column $\left(\alpha_{W C O}^{*}\right)$ gives the competitive ratio of the WCO mechanism (the optimal competitive ratio among all feasible mechanisms $(M, R)$ where $M$ allocates efficiently).

\begin{tabular}{|c||c|c||c|c||c|}
\hline & $\alpha_{D}^{*}$ & burn & $\alpha_{C}^{*}$ & burn & $\alpha_{W C O}^{*}$ \\
\hline $\mathrm{n}=4, \mathrm{~m}=1$ & 0.571 & 0 & 0.571 & 0 & 0.571 \\
\hline $\mathrm{n}=4, \mathrm{~m}=2$ & 0.286 & 1 & 0.667 & 0.67 & 0.250 \\
\hline $\mathrm{n}=4, \mathrm{~m}=3$ & 0.267 & 2 & 0.889 & 0.33 & 0 \\
\hline \hline $\mathrm{n}=6, \mathrm{~m}=1$ & 0.839 & 0 & 0.839 & 0 & 0.839 \\
\hline $\mathrm{n}=6, \mathrm{~m}=3$ & 0.410 & 1 & 0.800 & 0.60 & 0.375 \\
\hline $\mathrm{n}=6, \mathrm{~m}=5$ & 0.356 & 3 & 0.960 & 0.20 & 0 \\
\hline \hline $\mathrm{n}=8, \mathrm{~m}=1$ & 0.945 & 0 & 0.945 & 0 & 0.945 \\
\hline $\mathrm{n}=8, \mathrm{~m}=3$ & 0.646 & 0 & 0.762 & 0.71 & 0.646 \\
\hline $\mathrm{n}=8, \mathrm{~m}=5$ & 0.452 & 2 & 0.914 & 0.43 & 0.276 \\
\hline $\mathrm{n}=8, \mathrm{~m}=7$ & 0.422 & 4 & 0.980 & 0.14 & 0 \\
\hline
\end{tabular}

For the case of $n=10, m=1, \ldots, 9$, we compare the values of $\alpha_{W C O}^{*}, \alpha_{D}^{*}$ and $\alpha_{C}^{*}$ in Figure 1. When $m$ is small, the three values are the same. As $m$ gets large, the value of $\alpha_{W C O}^{*}$ decreases all the way to 0 ; the value of $\alpha_{D}^{*}$ also decreases but it gets stable when its value goes down to around 0.5 ; the value of $\alpha_{C}^{*}$ first decreases, but then increases, at the end almost reaches 1 .

Of course, $\alpha_{W C O}^{*} \leq \alpha_{D}^{*} \leq \alpha_{C}^{*}$; it turns out that all of these inequalities are sometimes strict. Therefore, in general we need to burn a random number of units to get the most competitive redistribution mechanism.

While we can use Theorem 1 in this way to solve for the

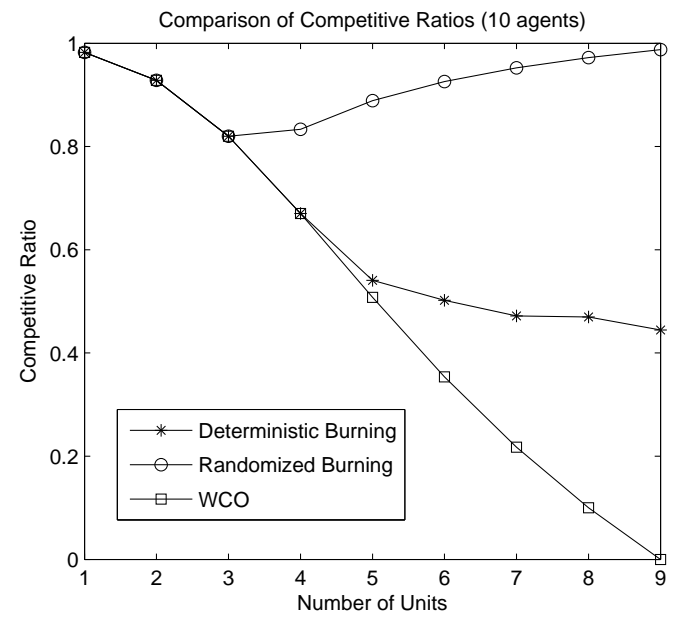

Figure 1: A comparison of $\alpha_{D}^{*}, \alpha_{C}^{*}$ and $\alpha_{W C O}^{*}$.

most competitive redistribution mechanism in this class of mechanisms for any given $n$ and $m$, it would be nice to have a general analytical characterization of the most competitive redistribution mechanism. The following claim specifies a burning-based redistribution mechanism for each $m, n$ pair, and gives the competitive ratio for these mechanisms. We conjecture that these mechanisms are in fact the most competitive in this class of mechanism (including the randomized mechanisms), but have not been able to prove it. (However, using the linear programming methodology from Theorem 1, we have verified that this conjecture is true for all $n \leq 10$.)

Claim 3. Given $n$ and $m$, using a redistribution mechanism $(M, R)$ where $M$ is a randomized burning allocation mechanism, we can achieve the following competitive ratio:

$$
\max \left\{1-\frac{\left(\begin{array}{c}
n-1 \\
m
\end{array}\right)}{\sum_{j=m}^{n-1}\left(\begin{array}{c}
n-1 \\
j
\end{array}\right)}, \frac{m n-n}{m n-m}\right\}
$$

If the first expression is greater (or equal), then the mechanism achieving the above ratio is the worst-case optimal $V C G$ redistribution mechanism (nothing is burned).

If the second expression is greater, then the mechanism achieving the above ratio is the following:

- Burn (throw away) one unit with probability $\frac{n-m}{n-1}$.

- The remaining units are allocated according to the VCG mechanism.

- After the VCG payments, every agent receives a redistribution payment of $\frac{m-1}{n-1}$ times the $m$-th highest bid among bids other than this agent's own bid. (Unlike the VCG payments, the redistribution does not depend on whether a unit was burned.)

Proof. We already know that the WCO mechanism is strategy-proof, feasible, and has competitive ratio $1-\frac{\left(\begin{array}{c}n-1 \\ m\end{array}\right)}{\sum_{j=m}^{n-1}\left(\begin{array}{c}n-1 \\ j\end{array}\right)}$. Hence, we only need to show that the other mechanism proposed in the claim is strategy-proof, feasible, and has competitive ratio $\frac{m n-n}{m n-m}$, for the values of $m, n$ for which this mechanism outperforms the WCO mechanism. 
If $m=1$, then $\frac{m n-n}{m n-m}=0$, which can not be greater than the competitive ratio of the WCO mechanism. So we only need to consider $m>1$. We have already proved that any randomized burning allocation mechanism is strategyproof. After introducing redistribution, the mechanism remains strategy-proof, because the redistribution does not depend on the agent's own bid. Individual rationality is satisfied because the randomized burning allocation mechanism is individually rational, and the redistribution is always nonnegative. For any bid profile $V$, the VCG revenue is $m v_{m+1}$ when nothing is burned, and the VCG revenue is $(m-1) v_{m}$ when one unit is burned. Together, the expected ${ }^{5}$ VCG revenue is $\frac{n-m}{n-1}(m-1) v_{m}+\left(1-\frac{n-m}{n-1}\right) m v_{m+1}=\frac{m-1}{n-1}\left(m v_{m+1}+\right.$ $\left.(n-m) v_{m}\right)$. For the agents bidding $v_{1}, \ldots, v_{m}$, the redistribution received is $\frac{m-1}{n-1} v_{m+1}$. For the other agents, the redistribution received is $\frac{m-1}{n-1} v_{m}$. Therefore, the total redistribution equals the total VCG payment, so the non-deficit criterion is satisfied. We conclude that the mechanism is feasible.

Now we show that the mechanism has competitive ratio $\frac{m n-n}{m n-m}$. With probability $\frac{n-m}{n-1}$, the total efficiency is $\sum_{i=1}^{m-1} v_{i}$ (one unit is burned). When nothing is burned, the total efficiency is $\sum_{i=1}^{m} v_{i}$. In expectation, the total efficiency is $\frac{n-m}{n-1} \sum_{i=1}^{m-1} v_{i}+\left(1-\frac{n-m}{n-1}\right) \sum_{i=1}^{m} v_{i}$. This is greater than or equal to $\frac{n-m}{n-1} \frac{m-1}{m} \sum_{i=1}^{m} v_{i}+\left(1-\frac{n-m}{n-1}\right) \sum_{i=1}^{m} v_{i}=$ $\frac{m n-n}{m n-m} \sum_{i=1}^{m} v_{i}$ (we have equality when all $v_{i}$ are equal). Since the total payment equals the total redistribution, efficiency is equal to welfare, so we conclude that we obtain the competitive ratio $\frac{m n-n}{m n-m}$.

Conjecture 1. The competitive ratio in Claim 3 is optimal for mechanisms $(M, R)$ where $M$ is a randomized burning allocation mechanism. That is, if $M$ is required to be a randomized burning allocation mechanism, then there is an optimal mechanism that either never burns anything (so that it coincides with the WCO mechanism), or burns exactly one unit with some probability, so that all the revenue can be redistributed.

\section{PARTITIONING UNITS AND AGENTS}

In this section, we study allocation mechanisms that are based on partitioning the units and the agents into groups. This is an idea that has previously been proven effective in mechanism design $[3,8]$. Based on this idea, we first characterize a class of strongly budget balanced allocation mechanisms (in the setting of multi-unit auctions with unit demand). Some of the mechanisms in this class have been

\footnotetext{
${ }^{5}$ The mechanism satisfies the non-deficit criterion only in expectation over the choice of whether to burn a unit. Alternatively, we can charge each agent her expected VCG payment, in which case there will certainly be no deficit. One may worry that this will result in individual rationality only holding in expectation. However, interestingly, individual rationality continues to hold unconditionally if we charge the expected VCG payment: the only agent that faces any randomness is the $m$ th agent, and she pays $\left(1-\frac{n-m}{n-1}\right) v_{m+1}$, but then receives a redistribution of $\frac{m-1}{n-1} v_{m+1}$, for a total payment of 0 , so that she is not unhappy even if the unit is thrown away. However, in this case, she would prefer to place a bid of 0 instead-so the resulting mechanism is strategy-proof only in expectation over the mechanism's random choice. (In contrast, the mechanism in Claim 3 is unconditionally strategy-proof and individually rational.)
}

proposed previously $[6,17]$. We focus on finding the most competitive mechanism in this class. Because all of the mechanisms in this class are strongly budget balanced, there will be no redistributions.

We start with two example mechanisms. They are both based on excluding one individual agent from the set of all agents. The first one is due to Moulin [17], and the second one is due to Faltings [6].

\section{Example Mechanism 1}

- Exclude one agent from the auction, uniformly at random.

- Assign one unit to the excluded agent at no charge.

- The remaining units are allocated to the remaining agents according to the VCG mechanism.

- Transfer all the VCG revenue to the excluded agent.

\section{Example Mechanism 2}

- Exclude one agent from the auction, uniformly at random.

- Units are allocated to the remaining agents according to the VCG mechanism.

- Transfer all the VCG revenue to the excluded agent.

Both example mechanisms are strategy-proof, individually rational and strongly budget balanced. In the first mechanism, one agent is excluded and assigned one unit. In the second mechanism, one agent is excluded and assigned zero units.

We now introduce our class of mechanisms that is based on partitioning the agents; this class generalizes both of the previous two mechanisms.

Definition 2. Given $n$ and $m$, for $n_{1} \in\left\{1, \ldots,\left\lfloor\frac{n}{2}\right\rfloor\right\}, m_{1} \in$ $\left\{0, \ldots, \min \left\{n_{1}, m\right\}\right\}$, we define the following mechanism:

- Pick $n_{1}$ agents to form one group, uniformly at random. The other $n-n_{1}$ agents form the second group.

- Allocate $m_{1}$ units among the first group, according to the VCG mechanism.

- Allocate the remaining $m-m_{1}$ units among the second group, according to the VCG mechanism.

- Transfer the VCG revenue from the first group to the second group, in any predetermined way.

- Transfer the VCG revenue from the second group to the first group, in any predetermined way.

We call this mechanism the $\left(n_{1}, m_{1}\right)$-partition mechanism.

We note that Example Mechanisms 1 and 2 are the $(1,1)$ partition mechanism and the (1,0)-partition mechanism, respectively.

ClaIM 4. The partition mechanisms are strategy-proof, individually rational, and strongly budget balanced. 
Proof. Without transferring the VCG revenue, every agent is participating in a VCG mechanism, which must be strategy-proof. For each agent, the transfer payment she receives depends only on the bids from the other group of agents, hence it does not affect her incentives. Therefore, the mechanisms are strategy-proof. Similarly, without transferring the VCG revenue, every agent is participating in a VCG mechanism, which must be individually rational. With transferring, the agents' utilities become higher or stay the same. Therefore, the mechanisms are individually rational. Finally, the strong budget balance property follows from the fact that the entire VCG revenue is transferred.

Since the partition mechanisms are strongly budget balanced, welfare must equal efficiency. Hence, for our objective of finding the most competitive partition mechanism, we can completely ignore the VCG payments and the revenue transferring process. That is, for the analysis that follows, we pretend that there are no payments of any kind; when we use the mechanism, we add the VCG payments and transfers back to achieve strategy-proofness. We now show that by ignoring the payments and transfers, the partition mechanisms become linear mechanisms (albeit linear mechanisms that are not strategy-proof, but this does not matter). Given $n$ and $m$, let $M_{n_{1}, m_{1}}$ be the $\left(n_{1}, m_{1}\right)$-partition mechanism. For any bid profile $V=\left(v_{1}, v_{2}, \ldots, v_{n}\right)$, under $M_{n_{1}, m_{1}}$, there is only a finite number of ways of dividing the $v_{i}$ into two groups of size $n_{1}$ and $n-n_{1}$ (and each of these ways receives equal probability). For any specific way of dividing, the agents' total efficiency is linear in the $v_{i}$. Since each way of dividing happens with equal probability, the expected total efficiency is also linear in the $v_{i}$. That is, $U_{M_{n_{1}, m_{1}}}(V)$ is linear in the $v_{i}$. We also have $P_{M_{n_{1}, m_{1}}}=0$. Hence, the partition mechanisms satisfy the linearity condition. The normalized individual rationality condition is also satisfied (after ignoring the VCG payments and the revenue transferring process). Thus, we can use the general technique in Theorem 1 to solve for the optimal partition mechanism. However, we now present a simpler solution technique based on the special structure of the class of partition mechanisms.

The following claim characterizes the competitive ratio of a given partition mechanism.

Claim 5. Given $n$ and $m$, the competitive ratio of the $\left(n_{1}, m_{1}\right)$-partition mechanism equals

$$
\frac{U_{M_{n_{1}, m_{1}}}(m)}{m}
$$

Here, $U_{M_{n_{1}, m_{1}}}(m)$ is the expected efficiency (welfare) under the $\left(n_{1}, m_{1}\right)$-partition mechanism when $m$ agents bid 1 and the remaining agents bid 0 . This competitive ratio is equal to

$$
\frac{\sum_{x \in X}\left(\begin{array}{c}
n_{1} \\
x
\end{array}\right)\left(\begin{array}{c}
n-n_{1} \\
m-x
\end{array}\right)\left(\min \left\{x, m_{1}\right\}+\min \left\{m-x, m-m_{1}\right\}\right)}{m\left(\begin{array}{c}
n \\
m
\end{array}\right)}
$$

Here, $X=\left\{x \mid 0 \leq x \leq n_{1}, 0 \leq m-x \leq n-n_{1}\right\}$. We will call this competitive ratio $\alpha_{n_{1}, m_{1}}^{*}$.

Proof. For bid profile $m$ (where $m$ agents bid 1 and the remaining agents bid 0 ), the perfect (omnipotent) mechanism would achieve an efficiency of $m$. Hence, $\frac{U_{M_{n_{1}}, m_{1}}(m)}{m}$ is an upper bound on $\alpha_{n_{1}, m_{1}}^{*}$.

We now show that $\frac{U_{M_{n_{1}}, m_{1}}(m)}{m}$ is equal to the second expression in the claim; then, we will show that $M_{n_{1}, m_{1}}$ does in fact attain this competitive ratio. In the $\left(n_{1}, m_{1}\right)$ partition mechanism, $n_{1}$ agents are randomly picked to form one group, and the remaining $n-n_{1}$ agents form a second group. If $m$ agents bid 1 and the remaining agents bid 0 , then the probability of having $x$ agents that bid 1 in the group of size $n_{1}$ is $\frac{\left(\begin{array}{c}n_{1} \\ x\end{array}\right)\left(\begin{array}{c}n-n_{1} \\ m-x\end{array}\right)}{\left(\begin{array}{c}n \\ m\end{array}\right)}$. The corresponding total welfare is $\left(\min \left\{x, m_{1}\right\}+\min \left\{m-x, m-m_{1}\right\}\right)$. The set of possible values of $x$ is $X$. It follows that $U_{M_{n_{1}, m_{1}}}(m)$ is equal to $\frac{\sum_{x \in X}\left(\begin{array}{c}n_{1} \\ x\end{array}\right)\left(\begin{array}{c}n-n_{1} \\ m-x\end{array}\right)\left(\min \left\{x, m_{1}\right\}+\min \left\{m-x, m-m_{1}\right\}\right)}{\left(\begin{array}{c}n \\ m\end{array}\right)}$.

All that is left to show is that $M_{n_{1}, m_{1}}$ does in fact attain this competitive ratio. Let us consider the following allocation mechanism, which is never better than $M_{n_{1}, m_{1}}$ :

- Pick $n_{1}$ agents to form one group, uniformly at random. The other $n-n_{1}$ agents form the second group.

- Remove the agents with the lowest $n-m$ bids.

- For the first group, if there are more than $m_{1}$ agents left, allocate $m_{1}$ units uniformly at random among the remaining agents in group one. Otherwise, allocate one unit to every remaining agent in group one.

- For the second group, if there are more than $m-m_{1}$ agents left, allocate $m-m_{1}$ units uniformly at random among the remaining agents in group two. Otherwise, allocate one unit to every remaining agent in group two.

For any bid profile, the above mechanism results in (weakly) lower efficiency than the $\left(n_{1}, m_{1}\right)$-partition mechanism, because in the partition mechanism, the units are assigned efficiently within each group, and in the modified mechanism they are not because of agent removal and random assignment.

Under the modified mechanism, only the agents bidding $v_{1}, \ldots, v_{m}$ possibly win any units, and the probability of winning is the same for each of them. For the bid profile in which $m$ agents bid 1 and the remaining agents bid 0 , the modified mechanism results in the same efficiency as the partition mechanism. Therefore, because in this case, a winning agent's utility is 1 , the expected number of winners under the modified mechanism is $U_{M_{n_{1}, m_{1}}}(m)$. But this probability must be the same for all bid profiles. So, using the fact that each of the top $m$ bidders is equally likely to win, for a general bid profile, the expected efficiency under the modified mechanism is $\frac{U_{M_{n_{1}}, m_{1}}(m)}{m} \sum_{i=1}^{m} v_{i}$; and we know that this is (weakly) lower than the expected efficiency under the $\left(n_{1}, m_{1}\right)$-partition mechanism. Hence, the $\left(n_{1}, m_{1}\right)$-partition mechanism has a competitive ratio of at least $\frac{U_{M_{n_{1}}, m_{1}}(m)}{m}$. We have already proved that $\frac{U_{M_{n_{1}}, m_{1}}(m)}{m}$ is an upper bound of $\alpha_{n_{1}, m_{1}}^{*}$, so this expression must be exactly equal to the competitive ratio.

So far, we have not considered mixtures over partition mechanisms. It could be that, by taking such mixtures, we can obtain more competitive mechanism. However, the following claim rules out the possibility of obtaining more competitive mechanisms by taking mixtures over partition mechanisms.

Claim 6. If $M$ is a mixture over $M_{1}, M_{2}, \ldots, M_{t}$, where the $M_{i}$ are partition mechanisms for different values of $n_{1}, m_{1}$, 
and $M_{i}$ is chosen with probability $p_{i}$, then there exists $1 \leq$ $j \leq t$ so that $M_{j}$ attains at least the competitive ratio of $M$.

ProOF. By the same argument as in Claim 5, the competitive ratio of $M$ is at most $\frac{U_{M}(m)}{m}$. We have that $\frac{U_{M}(m)}{m}=$ $\frac{\sum_{i=1}^{t} p_{i} U_{M_{i}}(m)}{m} \leq \max _{j} \frac{U_{M_{j}}(m)}{m}$. But $\frac{U_{M_{j}}(m)}{m}$ (where $j \in$ $\left.\arg \max _{j} \frac{U_{M_{j}}(m)}{m}\right)$ is the competitive ratio for $M_{j}$ by Claim 5 . Hence, $M_{j}$ is as competitive as $M$.

By Claim 5, for given $n$ and $m$, by maximizing

$$
\frac{\sum_{x \in X}\left(\begin{array}{c}
n_{1} \\
x
\end{array}\right)\left(\begin{array}{c}
n-n_{1} \\
m-x
\end{array}\right)\left(\min \left\{x, m_{1}\right\}+\min \left\{m-x, m-m_{1}\right\}\right)}{m\left(\begin{array}{c}
n \\
m
\end{array}\right)}
$$

over $n_{1}$ and $m_{1}$, we obtain the optimal $\left(n_{1}, m_{1}\right)$-partition mechanism. This mechanism is also optimal among all mixtures of partition mechanisms by Claim 6 . It would be nice to have a general analytical characterization of the optimal $n_{1}$ and $m_{1}$. The following conjecture specifies three partition mechanisms, and gives the corresponding competitive ratios. The conjecture states that for any $n$ and $m$, the optimal partition mechanism must be one of these three. Experimentally, we have verified that this conjecture is true for all $n \leq 10$.

ConjeCture 2. For any $n$ and $m$, the optimal partition mechanism is one of the following three:

$(1,0)$-partition mechanism, with competitive ratio $\frac{n-1}{n}$;

$(1,1)$-partition mechanism, with competitive ratio $\frac{n m+m-n}{n m}$;

$(2,1)$-partition mechanism, with competitive ratio

$$
\frac{\sum_{x \in X^{\prime}}\left(\begin{array}{c}
2 \\
x
\end{array}\right)\left(\begin{array}{c}
n-2 \\
m-x
\end{array}\right)(\min \{x, 1\}+\min \{m-x, m-1\})}{m\left(\begin{array}{c}
n \\
m
\end{array}\right)},
$$

where $X^{\prime}=\{x \mid 0 \leq x \leq 2,0 \leq m-x \leq n-2\}$.

In the following table, we present the results for various numbers of agents and units. The second column $\left(\alpha_{n_{1}, m_{1}}^{*}\right)$ gives the optimal competitive ratio among all partition mechanisms. The third column gives the values of $n_{1}$ and $m_{1}$, where the $\left(n_{1}, m_{1}\right)$-partition mechanism achieves the optimal competitive ratio.

\begin{tabular}{|c|c|c|}
\hline & $\alpha_{n_{1}, m_{1}}$ & $\left(n_{1}, m_{1}\right)$ \\
\hline$n=4, m=1$ & 0.750 & $(1,0)$ \\
\hline$n=4, m=2$ & 0.833 & $(2,1)$ \\
\hline$n=4, m=3$ & 0.917 & $(1,1)$ \\
\hline \hline$n=6, m=1$ & 0.833 & $(1,0)$ \\
\hline$n=6, m=3$ & 0.867 & $(2,1)$ \\
\hline$n=6, m=5$ & 0.967 & $(1,1)$ \\
\hline \hline$n=8, m=1$ & 0.875 & $(1,0)$ \\
\hline$n=8, m=3$ & 0.875 & $(1,0)$ \\
\hline$n=8, m=5$ & 0.925 & $(1,1)$ \\
\hline$n=8, m=7$ & 0.982 & $(1,1)$ \\
\hline
\end{tabular}

\section{GENERALIZED PARTITION MECHANISMS}

Finally, we slightly generalize the definition of partition mechanisms by allowing for empty groups of agents in the partition, as well as burning units.

Definition 3. Given $n$ and $m$, for nonnegative integers $n_{1}, n_{2}, m_{1}, m_{2}$ with $n_{1}+n_{2}=n, m_{1}+m_{2} \leq m$, we define the following mechanism:
- Pick $n_{1}$ agents to form one group, uniformly at random. The other $n-n_{1}$ agents form the second group. (One group can be empty.)

- Allocate $m_{1}$ units among the first group, according to the VCG mechanism.

- Allocate $m_{2}$ units among the second group, according to the VCG mechanism.

We call this mechanism the $\left(n_{1}, m_{1}, m_{2}\right)$-generalized partition mechanism.

We removed the transferring of VCG revenue from the definition, because when one group is empty, it is not possible to transfer to that group. However, we still allow for redistribution, so if both groups are nonempty (or, more generally, if we randomize only over generalized partition mechanisms in which both groups are nonempty) we will in fact redistribute all the VCG revenue.

The set of generalized partition mechanisms contains all the burning allocation mechanisms: the $\left(0,0, m_{2}\right)$-generalized partition mechanism is the mechanism in which $m-m_{2}$ units are burned, and the remaining units are allocated efficiently among all agents.

ClaIm 7. All generalized partition mechanisms are strategyproof and linear.

Proof. Every agent is participating in a VCG mechanism, which must be strategy-proof and individually rational. We also have that if an agent's bid is 0 , then her payment is 0 . Let $M$ be a generalized partition mechanism, $U_{M}$ and $P_{M}$ are the average of the efficiency and VCG revenue over all random partitions of the agents into groups of sizes $n_{1}$ and $n-n_{1}$. Given a specific way of partitioning, both the efficiency and the VCG revenue are linear in the $v_{i}$. Therefore, both $U_{M}$ and $P_{M}$ are linear in the $v_{i}$ as well.

We can now directly apply Theorem 1 to find the mechanism $(M, R)$ with the highest competitive ratio, given that $M$ is a mixture of the generalized partition mechanisms. In the following table, we present the results for various numbers of agents and units. The second column $\left(\alpha^{*}\right)$ gives the optimal competitive ratio among all $(M, R)$, under the constraint that $M$ is a mixture of the generalized partition mechanisms. The third column describes a mixture of generalized partition mechanisms that attains the optimal competitive ratio in each case (the meaning of $\left(n_{1}, m_{1}, m_{2}\right), p$ is that with probability $p$, we use the $\left(n_{1}, m_{1}, m_{2}\right)$-generalized partition mechanism). (We do not present the redistribution function because we do not know how to conveniently describe it in a table.)

\begin{tabular}{|c|c|c|}
\hline & $\alpha^{*}$ & allocation mechanism \\
\hline$n=4, m=1$ & 0.842 & $\begin{array}{l}(0,0,1), 0.37 \\
(1,0,1), 0.63\end{array}$ \\
\hline$n=4, m=2$ & 0.864 & $\begin{array}{l}(0,0,2), 0.18 \\
(2,1,1), 0.82\end{array}$ \\
\hline$n=4, m=3$ & 0.923 & $\begin{array}{l}(0,0,3), 0.08 \\
(1,1,2), 0.92\end{array}$ \\
\hline \hline$n=8, m=1$ & 0.962 & $\begin{array}{l}(0,0,1), 0.69 \\
(1,0,1), 0.31\end{array}$ \\
\hline$n=8, m=3$ & 0.908 & $\begin{array}{l}(0,0,3), 0.26 \\
(1,0,3), 0.74\end{array}$ \\
\hline$n=8, m=5$ & 0.928 & $(0,0,5), 0.04$ \\
& & $(1,1,4), 0.96$ \\
\hline$n=8, m=7$ & 0.982 & $(0,0,7), 0.02$ \\
& & $(1,1,6), 0.98$ \\
\hline
\end{tabular}




\section{CONCLUSION}

The VCG mechanism is not strongly budget balanced: in general, value flows out of the system of agents in the form of VCG payments, which reduces the agents' utilities. In many settings, the objective is to maximize the sum of the agents' utilities (taking payments into account). For this purpose, several VCG redistribution mechanisms have been proposed that redistribute a large fraction of the VCG payments back to the agents, in a way that maintains strategy-proofness and the non-deficit property. Unfortunately, sometimes even the best VCG redistribution mechanism fails to redistribute a substantial fraction of the VCG payments. This results in a low welfare for the agents, even though the items are allocated efficiently. In this paper, we studied strategy-proof allocation mechanisms that do not always allocate the items efficiently. It turns out that by allocating inefficiently, more payment can sometimes be redistributed, so that the net effect is an increase in the sum of the agents' utilities.

The objective that we pursued is to design mechanisms that are competitive in terms of welfare with the omnipotent perfect allocation. We defined linear allocation mechanisms. We proposed an optimization model for simultaneously finding an allocation mechanism and a payment redistribution rule which together are optimal, given that the allocation mechanism is required to be either one of, or a mixture of, a finite set of specified linear allocation mechanisms. Finally, we proposed several specific (linear) mechanisms that are based on burning units, excluding agents, and (most generally) partitioning the units and agents into groups. We showed or conjectured that these mechanisms are optimal among various classes of mechanisms.

We have assumed in this paper that random bits are not chosen adversarially, that is, the worst-case adversary controls the bidders' valuations but not our random choices. If the adversary also controls our random choices (which would correspond to a different notion of worst-case optimality), then it never hurts to use a deterministic mechanism. To illustrate how this affects our results, let us consider Example Mechanism 2 of Section 6, in which we (uniformly) randomly choose one agent to be excluded from the auction and assign this agent zero units. This mechanism is competitive in the sense of this paper. (An agent is excluded with probability $\frac{1}{n}$, hence the competitive ratio is $\frac{n-1}{n}$.) However, if we use the alternative notion of worst-case optimality, then the bidder with the highest valuation will always be excluded, so that the mechanism is not competitive. Under this alternative notion, among all maximal-in-range allocation mechanisms, either Example Mechanism 1 of Section 6 or the worst-case optimal mechanism is an optimal mechanism, for the following reasons. If, in an optimal maximal-in-range mechanism, there is some subset of $m$ agents that cannot all win at the same time, then the competitive ratio is at most $\frac{m-1}{m}$, and we can achieve this competitive ratio using Example Mechanism 1. On the other hand, if any subset of $m$ agents can win at the same time, then the mechanism must be efficient, and it can do no better than the worst-case optimal mechanism.

Future research on maximizing agents' welfare under the constraint of strategy-proofness can take a number of directions. First of all, we have left several open questions. Other questions include the following. Are there other families of inefficient mechanisms that result in a high welfare for the agents? Among what more general classes of mechanisms are the mechanisms proposed in this paper optimal? Can we generalize some of these results to wider settings, for example, settings without unit demand, or even combinatorial auctions? What happens if we change the objective, for example, if we have a prior distribution over bid profiles and we wish to maximize expected welfare?

\section{REFERENCES}

[1] G. Aggarwal, A. Fiat, A. Goldberg, J. Hartline, N. Immorlica, and M. Sudan. Derandomization of auctions. In Proceedings of the Annual Symposium on Theory of Computing (STOC), pages 619-625, 2005.

[2] M. J. Bailey. The demand revealing process: to distribute the surplus. Public Choice, 1997.

[3] M.-F. Balcan, A. Blum, J. D. Hartline, and Y. Mansour. Mechanism design via machine learning. In Proceedings of the Annual Symposium on Foundations of Computer Science (FOCS), pages 605-614, 2005.

[4] R. Cavallo. Optimal decision-making with minimal waste: Strategyproof redistribution of VCG payments. In $A A M A S$ 2006, pages 882-889, Hakodate, Japan.

[5] E. H. Clarke. Multipart pricing of public goods. Public Choice, 11:17-33, 1971.

[6] B. Faltings. A budget-balanced, incentive-compatible scheme for social choice. In Agent-Mediated Electronic Commerce (AMEC), LNAI, 3435, pages 30-43, 2005.

[7] J. Feigenbaum, C. H. Papadimitriou, and S. Shenker. Sharing the cost of muliticast transmissions. Journal of Computer and System Sciences, 63:21-41, 2001.

[8] A. Goldberg, J. Hartline, A. Karlin, M. Saks, and A. Wright. Competitive auctions. Games and Economic Behavior, 2006.

[9] J. Green and J.-J. Laffont. Characterization of satisfactory mechanisms for the revelation of preferences for public goods. Econometrica, 1977.

[10] J. Green and J.-J. Laffont. Incentives in Public Decision Making. Amsterdam: North-Holland, 1979.

[11] T. Groves. Incentives in teams. Econometrica, 1973.

[12] M. Guo and V. Conitzer. Worst-case optimal redistribution of VCG payments. In $E C 2007$, pages 30-39, San Diego, CA, USA.

[13] M. Guo and V. Conitzer. Optimal-in-expectation redistribution mechanisms. In $A A M A S$ 2008, Estoril, Portugal. To appear.

[14] M. Guo and V. Conitzer. Undominated VCG redistribution mechanisms. In $A A M A S$ 2008, Estoril, Portugal. To appear.

[15] J. Hartline and R. McGrew. From optimal limited to unlimited supply auctions. In EC 2005, pages 175-182, Vancouver, Canada.

[16] L. Hurwicz. On the existence of allocation systems whose manipulative Nash equilibria are Pareto optimal, 1975. Presented at the 3rd World Congress of the Econometric Society.

[17] H. Moulin. Efficient, strategy-proof and almost budget-balanced assignment, March 2007. Working Paper.

[18] R. Myerson and M. Satterthwaite. Efficient mechanisms for bilateral trading. Journal of Economic Theory, 28:265-281, 1983.

[19] D. Parkes, J. Kalagnanam, and M. Eso. Achieving budget-balance with Vickrey-based payment schemes in exchanges. In IJCAI 2001, Seattle, WA.

[20] W. Vickrey. Counterspeculation, auctions, and competitive sealed tenders. Journal of Finance, $16: 8-37,1961$. 